\title{
Importance of Alpha Lipoic Acid (ALA): Antidiabetic and Antioxidant Effects
}

\section{Anna Votano | Alessia Bonofiglio | Federica Catalano | Claudia Paone*}

*Correspondence: Claudia Paone

Address: University of Studies “Magna Græcia” of Catanzaro, Italy

e-mail $\bowtie$ : cla.31@libero.it

Received: 15 March 2021; Accepted: 22 March 2021

Copyright: (C) 2021 Votano A. This is an open-access article distributed under the terms of the Creative Commons Attribution License, which permits unrestricted use, distribution, and reproduction in any medium, provided that the original work is properly cited.

\section{ABSTRACT}

Within our organism, during the numerous metabolic reactions, free radicals (or ROS) are formed, unstable molecules that contain one or more unseated electrons and are responsible for oxidative stress. The organism must cope with and eliminate these free radicals that can create indiscriminate damage on biological macromolecules. To counteract their action and prevent cellular oxidation, the presence of molecules defined as antioxidants is fundamental. Some studies have seen that oxidative stress, caused by the presence of free radicals, is related to the onset of cardiovascular disease, diabetes, tumors and other chronic diseases.

Alpha lipoic acid (ALA) is an acid know for its positive effects on health, in particular its antidiabetic and antioxidant effects, and its effects on weight loss. Given its properties, in this study we want to explain in detail the mechanisms that determine these effects, also based on what is found in the literature. Objective: Analyze the importance of alpha lipoic acid and its antidiabetic and antioxidant effects.

Design: We have based the study in a bibliographic review.

Result: The antioxidant effect is due to its ability to neutralize free radicals and oxygen reactive species (ROS) and the ability to enhance the action of other endogenous antioxidants, such as vitamin $\mathrm{E}$ and vitamin $\mathrm{C}$. The antidiabetic effect is to improve the effectiveness of insulin and the transport of glucose into cells, thus leading to a reduction in insulin resistance. Conclusions: With this study we have demonstrated in detail the main mechanisms by which ALA acts and determines its antioxidant and antidiabetic effects. Our body is able to defend itself against ROS thanks to the presence of natural antioxidant substances, but sometimes these are not enough, so it is necessary to take supplements. It should be remembered that ALA is not a medicine and has no healing properties but its integration can be useful for the well-being of the organism.

Keywords: Alpha Lipoic Acid, Diabetes, Antioxidant Effects, Antidiabetic Effects

\section{Introduction}

Alpha lipoic acid (ALA), also known as thioptic acid, is a fatty acid synthesized by the body from the amino acid cysteine. It was first discovered in 1951 from bovine liver extracts, by American biochemists L.J. Reed and I.C. Gunsalus. Until the 1980s it was considered a vitamin, and only later as an antioxidant. It was then discovered that it can be synthesized in the human body, a the mitochondrial level, starting 
from actane acid and L-cysteine (Uchida et al., 2015). From a structural point of view it is a small amphipathic molecule, formed by a chain of eight carbon atoms, two of oxygen in the carboxylic group and two of sulfur located in the terminal part (Fig. 1). Its use in clinical practice dates back to the 1970s thanks to Burt Berkson who injected ALA in patients with fulminant hepatitis due to mushroom poisoning and about two weeks after treatment, liver function was back to normal. In the same period he used ALA in animal models of diabetes and from the observations that emerged from these experimental studies it was deduced that ALA could be useful in diabetic neuropathy as it significantly improved painful symptoms. This often painful condition is caused in part by the diabetic complications that arise when diabetics have not kept their diabetes under control (Andreea Rotaru, 2020; Agathos et al., 2018).

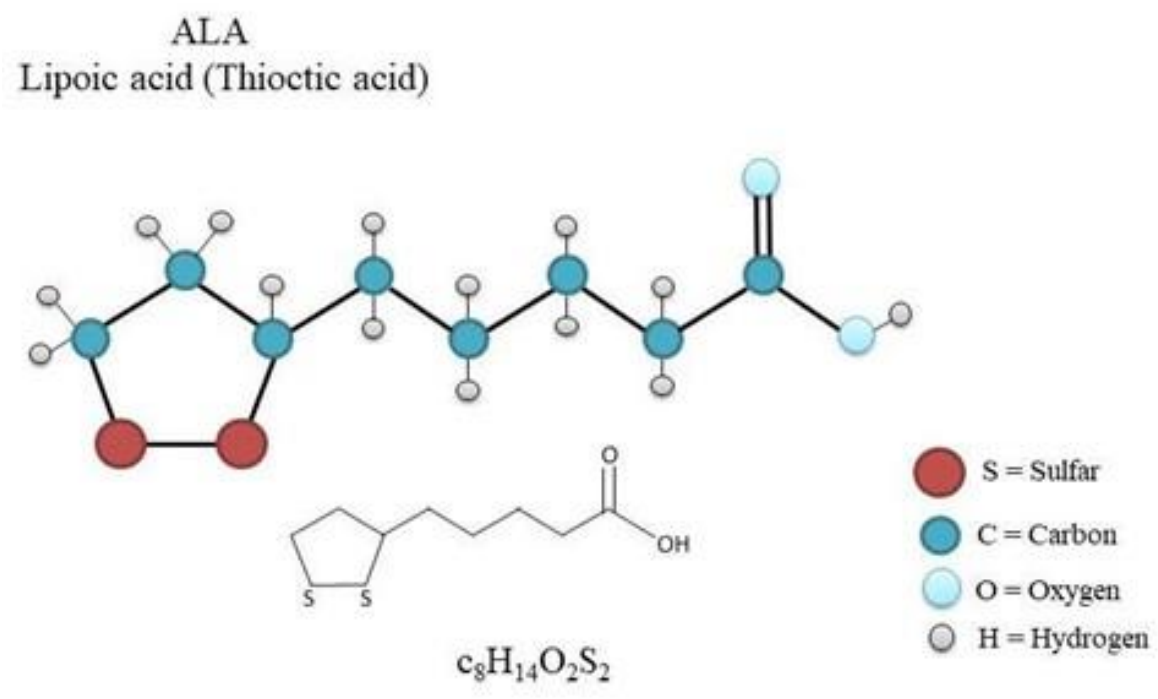

Figure 1: Structure of alpha lipoic acid. Chemically it is a small molecule, consisting of a chain of eight carbon atoms and two sulfar atoms located in the terminal part

In addition to diabetes, another chronic disease is obesity. According to a recent meta-analysis, Suat $\mathrm{K}$. and colleagues, found that the overall weight loss was $1.27 \mathrm{~kg}(\mathrm{CI}=-2,29$ to $-0,25)$ greater in treatment with ALA than in the placebo group (Kucukgoncu et al., 2018). Eun H.K. and colleagues found that intake of $1800 \mathrm{mg} /$ day of ALA led to modest weight loss in obese subjects (Koh et al., 2011). Bradley C. and other authors have shown in Zucker rat models that ALA has protective effects against hypercholesterolemia and the accumulation of hepatic fat in conditions of overeating and in case of genetic predisposition. Therefore, the integration of ALA, reduces plasma cholesterol probably throught a mechanism dependent on PCSK (Carrier et al., 2014).

Structure of alpha lipoic acid. Chemically it is a small molecule, consisting of a chain of eight carbon atoms and two sulfar atoms located in the terminal part. 
ALA exists in nature as an oxidized form (cyclic disulfide) and as a reduced form (dihydrolipoic acid- DHLA) (Fig. 2); the reduced form presents the two sulfur atoms in free thiol form (-SH), while the oxidized form presents a disulfide bridge (-S-S) which generates a dithiolane ring. Furthermore, the two forms are interconvertible throught redox reactions (Solmonson and DeBerardinis, 2018). Its particular structure allows the ALA to intervene in oxidation reduction reactions and to act as a biological carrier of electrons or acetyl groups.
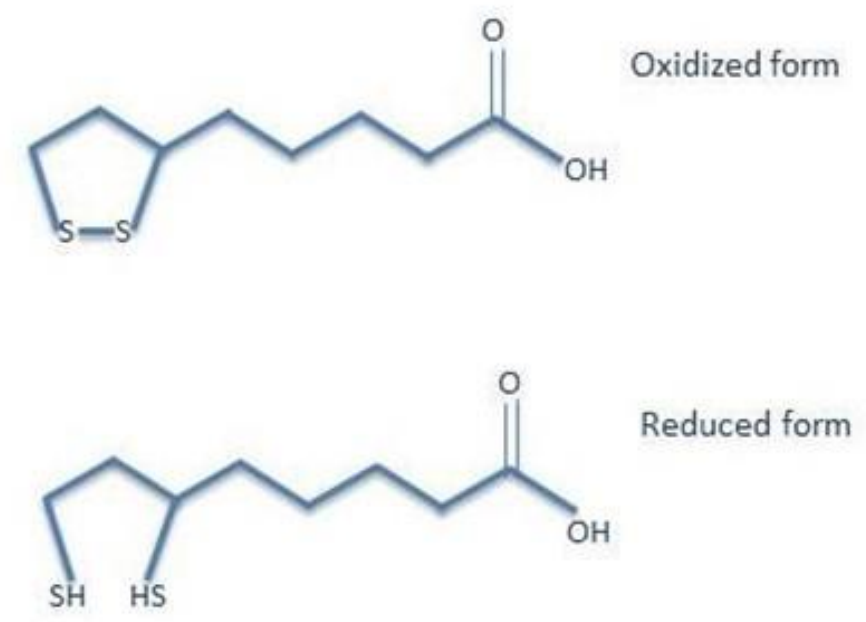

Figure 2: ALA exists in nature as an oxidized form (cyclic disulfide) and as a reduced form (dihydrolipoic acid- DHLA)

ALA, being fat soluble, that is, it can be distributed in all parts of the body, has the ability to cross the blood-brain and hematoretinal barrier, exerting its effects also at the ocular, cerebital and nervous system levels (Gomes and Negrato, 2014; Ciudin et al., 2017). We find it in foods such as: red meats, offal, potatoes, broccoli, peas, brussels sprouts, carrots, beets and spinach (Gorąca et al., 2011).

Once ingested, it is absorbed in the intestine and from there it reaches the liver through the portal circulation, to be then distributed to the various tissues of the body. It follows that a healthy diet, which includes meat and vegetables, is an excellent source of ALA but sometimes the amount of ALA in diet is not enough to satisfy our body's needs.

Our body is able to synthesize small quantities and then decrease as the body undergoes aging. In order to receive the right quantities to have its beneficial effects, it is recommended to take ALA through supplements. It should be remembered that food intake reduces its bioavailability, therefore it is recommended to take it between meals, at least 2 hours after a meal. Another reason fot taking ALA between meals is due to the fact that the stomach $\mathrm{pH}$ favors the absorption of ALA, as it does not compete with other nutrients for absorption (Brufani and Figliola, 2014). 
The recommended daily dose is $300-600 \mathrm{mg}$, preferably in combination with other antioxidants (glutathione, vitamin C, vitamin E, selenium, coenzyme Q10, etc.) and with the B vitamins. Some companies offend extended release formulations of ALA, particularly suitable for diabetics. It has been seem that ALA brings many benefits and is well tolerated, but great care must be taken as, if taken at higher doses than those indicated, they can cause hypoglycemia, migraines, muscle cramps, paraesthesia (Salehi et al., 2019; Mendoza-Núñez et al., 2019; Derosa et al., 2016; Fogacci et al., 2020).

\section{Antioxidant Effect of Alpha Lipoic Acid}

When we feed on macromolecules they are consumed through numerous oxidation reactions. These reactions can lead to the formation of free radicals, as the oxygen used in these metabolic processes to produce energy can give rise to the formation of free radicals or "Reactive Oxygen Radicals" (ROS). These free oxygen radicals are highly reactive and unstable molecules that normally form in small amounts in cellular aerobic metabolism. Reactive and unstable means that they have an unseated electron in their outfielder orbital that is very reactive and tends to subtract from the molecules, with which it comes into contact, the electron it needs to complete its external orbital and became stable (Fig. 3).

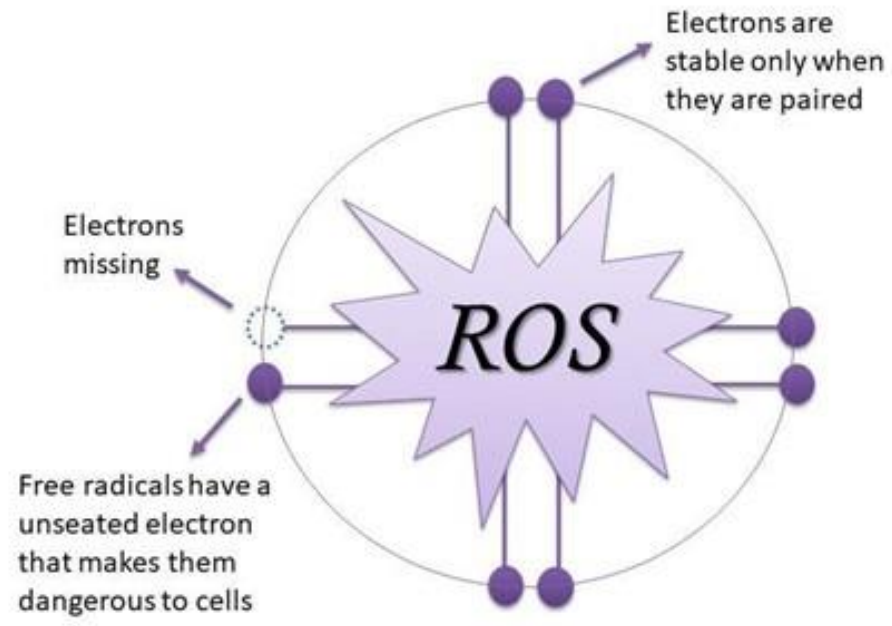

Figure 3: Reactive Oxygen Radicals" (ROS)

Free radicals are responsible for destructive action against cells or certain cellular components. It is now known that free radicals are involved in the process of tissue again and in the development of numerous chronic and degenerative diseases (Schmidt et al., 2015; Ergul Belge Kurutas, 2016).

Our organisms able to defend itself from the presence of free radicals by synthesizing substances called antioxidants. Antioxidants are chemicals (molecules, ions or radicals) or physical agents that slow down or prevent the oxidation of other substances, as they have the task of providing free radicals with the electrons they lack (Kasote et al., 2015). Antioxidants can be divided into endogenous and exogenous 
depending on whether the body is able to synthesize them or if they must be introduced with food. Endogenous antioxidants constitute the first line of defense of the organism. These may be insufficient, so the intake of exogenous antioxidants is important in order to fully meet the needs of the organism. Exogenous antioxidants are non-enzymatic in nature and are found almost exclusively in foods of plant origin (Ghibu et al., 2009).

Antioxidants are present in sufficient quantities in a balanced and complete diet. There are pathological and non-pathological situations, in which the production of these ROS is such that the antioxidant capacities are insufficient and particular condition is established indicated as oxidative stress. In these conditions, high levels of ROS can be found, so it is recommended to favor the intake of foods particularly rich in antioxidants and/or to take antioxidant-based supplements (Salehi et al., 2018; Halliwell and Gutteridge, 1990).

Molecules that react with free radicals become unstable and search for an electron, generating radicals that can propagate the chain reaction generated by the initiator radical. The radical, once formed, are able to alter some metabolic activities and some cellular structures. The uncontrolled production of free radicals is a factor that contributes to the onset of many disorders. ALA, especially in recent years, has been used as a food additive, in order to give beneficial effects in the management and treatment of various types of disorders, such as: endothelial dysfunction, atherosclerosis, diabetes mellitus, degenerative diseases and other (El Barky et al., 2017).

It is established that there is a close relationship between endothelial dysfunction and common cardiovascular risk factors, such as diabetes mellitus, arterial hypertension, hypercolesterolemia and aging. This is because free radicals, generated by pro-inflammatory situations and oxidative stress, favor the formation of endothelin ET-1, a powerful biological vasoconstrictor that is synthesized de novo by endothelial cells following stimulation by mediators, such as: IL-1, adrenaline, insulin, vasopressin (Van Houtte and Mombouli, 1996; Rossant and Howard, 2002). ALA can block Endothelin-1 receptors (AT1), improving and repairing endothelial function and reducing the formation of atherosclerotic plaque, influencing cardiovascular risk (CVD) through beneficial actions on LDL oxidation and plaque formation. In addition, ALA reduces the risk of atherosclerosis because it reduces he levels of inflammatory cytokines in the blood and therefore reduces the changes in vascular cells and normalizes blood pressure (Neurodiab, 2018; Biewenga et al., 1997).

According to some studies, ALA has a strong antioxidant action with the characteristic of being active, especially in its reduced form, against free radicals both in the aqueous comportment (cytoplasm) and in the lipid compartment (membranes) of the cells. Its reduced form, dihydrolipoic acid (DHLA) has 
antioxidant pharmacological properties, as it is able to destroy free radicals and attenuates the release of free radicals and cytotoxic cytokines, as ALA has an influence on the second nuclear transcription factor (NF-kB, factor) (Anna Goraca, 2011).

\section{Antidiabetic Effects of Alpha Lipoic Acid}

According to several studies, it has been shown that ALA can contribute to the control of diabetes, especially type 2 diabetes mellitus (T2DM). T2DM is a metabolic disorder characterized by inadequate insulin secretion and insulin resistance. Hyperglycemia can increase the production of free radicals and inflammatory cytokines (Su et al., 2013; Papachristoforou et al., 2020). ALA improves the effectiveness of insulin and the transport of glucose into the cells and reduces insulin resistance (Oluwafemi, 2019). Several studies have shown that the use of ALA has beneficial effects thanks to its ability to increase both the absorption of sugar in insulin-sensitive and insulin-resistant muscle tissues and to stimulate the glucose uptake by the repartition of glucose transporters to the plasma membrane, and tyrosine phosphorylation of insulin receptor substrate-1 (Fig. 4). As D. Konrad and colleagues state, the cofactor of mitochondrial dehydrogenase complexes and potent antioxidant alpha lipoic acid has been shown to lower blood glucose in diabetic animals. ALA enhances glucose uptake and GLUT1 and GLUT4 translocation in 3T3-L1 adipocytes and L6 myotubes, mimicking insulin action (Konrad et al., 2001).

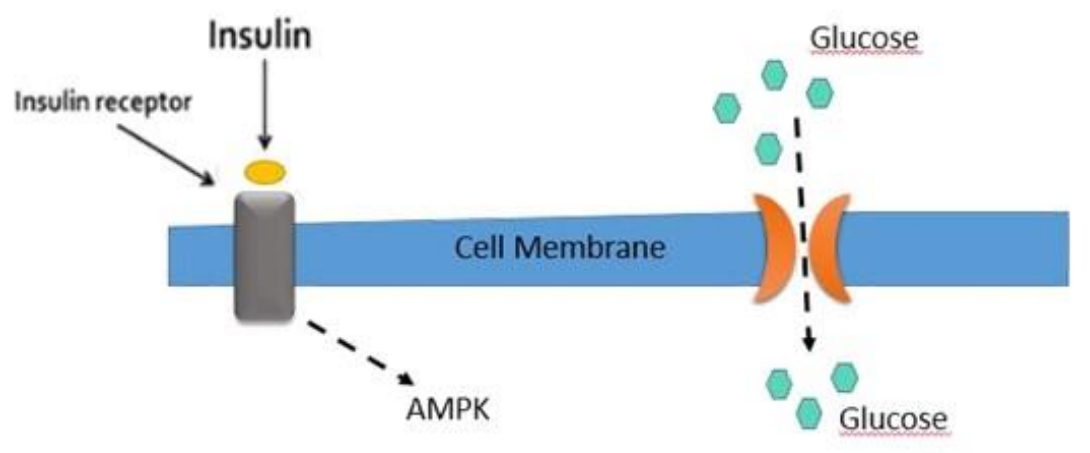

Figure 4: ALA has beneficial effects thanks to its ability to increase both the absorption of sugar in insulin-sensitive and insulinresistant muscle tissues and to stimulate the glucose uptake by the repartition of glucose transporters to the plasma membrane

Furthermore, Konrad and colleagues have shown that ALA is able to increase the absorption of glucose in insulin-sensitive and insulin-resistant muscle tissues because it stimulates the protein kinase AMPK, which increases the absorption of glucose into the cell ALA protects the nerves by limiting the damage caused by free radicals, improving the speed of nerve communication and normalizing nervous sensitivity. ALA can be useful in peripheral neuropathies, in particular in diabetic neuropathy, in which it significantly improves painful symptoms (Amara et al., 2019; Mijnhout et al., 2012; Mrakic-Sposta et al., 2018). 
ALA fights free radicals in every part of the neuron and is the only antioxidant that can do so. The peculiarity of ALA is that it acts both inside the cell, in an aqueous environments, and in an oily environment, that is, rich in lipids such as the membrane of neurons (Tutelyan et al., 2019).

Therefore, ALA can reach all the comportments of a cell, protecting it inside and outside from the attack of free radicals (or ROS) (Fig. 5).

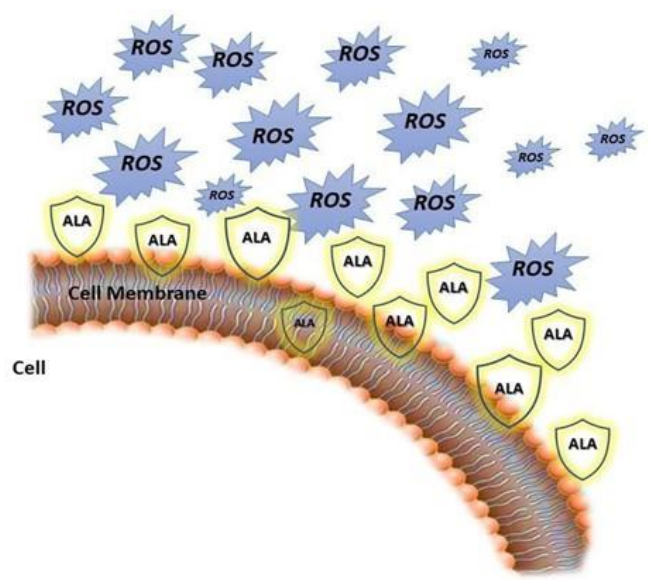

Figure 5: ALA can reach all the comportments of a cell, protecting it inside and outside from the attack of free radicals (or ROS)

As can be seen in the figure, ALA exerts an antioxidant action through the so-called "scavenger" action on ROS. Furthermore, ALA plays an important role in cellular energy metabolism, acting as a cofactor for numerous enzymes that participate in the process of converting glucose, fatty acids and other energy sources into ATP. It is possible that free radicals (or ROS) can attack and damage the membranes of the mitochondria, sites of energy resources and nerve self-repair. ALA can also recycle and regenerate endogenous antioxidants, such as: vitamin C, vitamin E, coenzyme $Q$ and glutathione. Doing so increases its effects and protects nerves and bloos vessels from ROS attacks. A 2012 study by Mijhout GS states that 3-week treatment at a daily dosage of $600 \mathrm{mg}$ intravenous ALA significantly reduces neuropathic pain in diabetic patients (Ziegler, 2011; Perez-Matos et al., 2017).

\section{Conclusions}

ALA is a fatty acid synthesized by the body or even taken by supplements. Lately, especially among young people who attend gyms, there is often talk of alpha lipoic acid, as it helps the loss of body weight. Our study aims to demonstrate, according to the studies in the literature, that ALA has other important effects. Thanks to its antioxidant and antidiabetic properties, according to several studies, the integration of ALA can be userful in case of diabetes, ALA supplementation should be used with caution when administered together with hypoglycemic, as ALA has a hypoglycemic effect. It will take even more scientific evidence to demonstrate the clinical efficacy of alpha lipoic acid intake. 


\section{Acknowledgement: The authors have no conflicts of interest to report.}

\section{References}

Agathos E, Tentolouris A, Eleftheriadou I, Katsaouni P, Nemtzas I, Petrou A, Papanikolaou C, Tentolouris N. Effect of $\alpha$ lipoic acid on symptoms and quality of life in patients with painful diabetic neuropathy. J Int Med Res 2018; 46: 1779-1790.

Amara F, Hafez S, Orabi A, El Etriby A, Rahim AA, Zakaria E, Koura F, Talaat FM, Gawish H, Attia I, Aziz MF. Review of Diabetic Polyneuropathy: Pathogenesis, Diagnosis and Management According to the Consensus of Egyptian Experts. Curr Diabetes Rev 2019; 15: 340-345.

Andreea Rotaru. The Neuroprotective role of alpha thioctic acid and vitamin B complex in diabetic neuropathy - an experimental study. Curr Health Sci J 2020; 46: 150-155.

Anna Goraca. Lipoic acid-biological activity and therapeutic potential. Pharmacological Reports 2011; 63: 849-858.

Biewenga GP, Haenen GR, Bast A. The pharmacology of the antioxidant lipoic acid. Gen Pharmacol 1997; 29: $315-331$.

Brufani M and Figliola R. (R)- $\alpha$-lipoic acid oral liquid formulation: pharmacokinetic parameters and therapeutic efficacy. Acta Biomed 2014; 85: 108-115.

Carrier B, Wen S, Zigouras S, Browne RW, Li Z, Patel MS, Williamson DL, Rideout TC. Alpha-lipoic acid reduces LDLparticle number and PCSK9 concentrations in high-fat fed obese zucker rats. PLoS One 2014; 9: e90863.

Ciudin A, Simó-Servat O, Hernández C, Arcos G, Diego S, Sanabria Á, Sotolongo Ó, Hernández I, Boada M, Simó R. Retinal Microperimentry: A New Tool for Identifyng Patients with Tyoe 2 Diabetes at Risk for Developing Alzheimer Disease. Diabetes 2017; 66: 3098-3104.

Derosa G, D’Angelo A, Romano D, Maffioli P. A Clinical Trial about a food supplement containing $\alpha$-Lipoic Acid on Oxidative Stress markers in Type 2 Diabetic Patients. Int J Mol Sci 2016; 17: 1802. id1016.

El Barky AR, Hussein SA, Mohamed TM. The potent antioxidant alpha lipoic acid. J Plant Chem and Ecophysiology 2017; 2:

Ergul Belge Kurutas. The importance of antioxidants which play the role in cellular response against oxidative/nitrosative stress: current state. Nutr J 2016; 15: 71.

Fogacci F, Rizzo M, Krogager C, Kennedy C, Georges CM, Knežević T, Liberopoulos E, Vallée A, Pérez-Martínez P, Wenstedt EF, Šatrauskienė A. Safety evaluation of $\alpha$-lipoic acid supplementation: a systematic review and meta-analysis of randomized placebo-controlled clinical studies. Antioxidants (Basel) 2020; 9: 1011.

Ghibu S, Richard C, Vergely C, Zeller M, Cottin Y, Rochette L. Antioxidant properties of an endogenous thiol: alpha-lipoic acid, useful in the prevention of cardiovascular disease. J Cardiovascular Pharmacol 2009; 54: 391-398

Gomes MB and Negrato CA. Alpha-lipoic acid as a pleiotropic compound with potential therapeutic use in diabetes and other chornic diseases. Diabetol Metab Syndr 2014; 6: 1-8.

Gorąca A, Huk-Kolega H, Piechota A, Kleniewska P, Ciejka E, Skibska B. Lipoic acid - biologic-l activity and therapeutic potential. Pharmacol Rep 2011; 63: 849-858.

Halliwell B and Gutteridge JM. The antioxidants of human extracellular fluids. Arch. Biochem. Biophys 1990; $208:$ 1-8.

Kasote DM, Katyare SS, Hegde MV, Bae H. Significance of antioxidant potential of plants and its relevance to therapeutic applications. Int J Biol Sci 2015; 11: 982-991.

Koh EH, Lee WJ, Lee SA, Kim EH, Cho EH, Jeong E, Kim DW, Kim MS, Park JY, Park KG, Lee HJ. Effects of alpha-lipoic acid on body weight in obese subjects. Am J Med 2011; 124: 85.e1-8.

Konrad D, Somwar R, Sweeney G, Yaworsky K, Hayashi M, Ramlal T, Klip A. The antihyperglycemic drug alpha-lipoic acid 
stimulates glucose uptake via both GLUT4 translocation and GLUT4 activation: potential role of p38 mitogen-activated protein kinase in GLUT4 activation. Diabetes 2001; 50: 1464-1471.

Kucukgoncu S, Zhou E, Lucas KB, Tek C. Alpha-lipoic acid (ALA) as a supplementation for weight loss: results from a metaanalysis of randomized controlled trials. Obes Rev 2018; 18: 594-601.

Mendoza-Núñez VM, García-Martínez BI, Rosado-Pérez J, Santiago-Osorio E, Pedraza-Chaverri J, Hernández-Abad VJ. The effect of $600 \mathrm{mg}$ aplpha-lipoic acid supplementation on oxidative stress, inflammation, and RAGE in older adults with type 2 diabetes mellitus. Oxid Med Cell Longev 2019; 2019: 3276958.

Mijnhout GS, Kollen BJ, Alkhalaf A, Kleefstra N, Bilo HJ. Alpha lipoic acid for symptomatic peripheral neuropathy in patients with diabetes: a meta-analysis of randomized controlled trials. Int J Endocrinol 2012; 2012: 456279

Mrakic-Sposta S, Vezzoli A, Maderna L, Gregorini F, Montorsi M, Moretti S, Greco F, Cova E, Gussoni M. R (+) - Thioctic Acid Effects on Oxidative Stress and Peripheral neuropathy in type II diabetic patients: preliminary results by elctron paramagnetic resonance and electroneurography. Oxid Med Cell Longev 2018; 2018: 1767265.

Neurodiab. Rome, 4-7 September 2018. Scientific Program \& Abstract Book, 2018.

Oluwafemi 00. Type 2 diabetes mellitus, oxidative stress and inflammation: examining the links. Int J Physiol Pathophysiol Pharmacol 2019; 11: 45-63.

Papachristoforou E, Lambadiari V, Maratou E, Makrilakis K. Association of glycemic indices (hyperglycemia, glucose variability, and hypoglycemia) with oxidative stress and diabetic complications. J Diabetes Res 2020; 2020: 7489795.

Perez-Matos MC, Morales-Alvarez MC, Mendivil CO. Lipids: A Suitable Therapeutic Target in Diabetic Neuropathy? J Diabetes Res 2017; 2017: 6943851.

Rossant J and Howard L. Signaling pathways in vascular development. Ann Rev Cell Dev Biol 2002; $18: 541$.

Salehi B, Berkay Yılmaz Y, Antika G, Boyunegmez Tumer T, Fawzi Mahomoodally M, Lobine D, Akram M, Riaz M, Capanoglu E, Sharopov F, Martins N. Insight on the Use of $\alpha$-Lipoic Acid for Therapeutic Purposes. Biomolecules 2019; 9: 356.

Salehi B, Martorell M, Arbiser JL, Sureda A, Martins N, Maurya PK, Sharifi-Rad M, Kumar P, Sharifi-Rad J. Antioxidants: positive or negative actors? Biomolecules 2018; 8: 124.

Schmidt HH, Stocker R, Vollbracht C, Paulsen G, Riley D, Daiber A, Cuadrado A. Antioxidants in translation medicine. Antioxid Redox Signal 2015; 23: 1130-1143.

Solmonson A and DeBerardinis RJ. Lipoic acid metabolism and mitochondrial redox regulation. J Biol chem 2018; 293: 7522-7530.

Su H, Ji L, Xing W, Zhang W, Zhou H, Qian X, Wang X, Gao F, Sun X, Zhang H. Acute hyperglycaemia enhances oxidative stress and aggravates myocardial ischaemia/reperfusion injury: role of thioredoxin-interacting protein. J Cell Mol Med 2013; 17: 181-191.

Tutelyan VA, Makhova AA, Pogozheva AV, Shikh EV, Elizarova EV, Khotimchenko SA. Lipoic acid: physiological role and prospects for clinical application. Vopr Pitan 2019; 88: 6-11.

Uchida R, Okamoto H, Ikuta N, Terao K, Hirota T. Enantioselective Pharmacokinetics of $\alpha$-Lipoic acid in rats. Int J Mol Sci 2015; 16: 22781-22784.

Van Houtte PM and Mombouli JV. Vacular endothelium: vasoactive mediators. Progress Cardiovascular Diseases 1996; 39: 229.

Ziegler D. Efficacy and safety of antioxidant treatment with $\alpha$-lipoic acid over 4 years in diabetic polyneuropathy. Diabetes Care 2011; 34: 2054-2060. 